\title{
Emergence of Objective Reality in an Irreversible Friend Thought Experiment
}

\author{
Robert L. Shuler \\ Shuler Research, Former NASA, Houston, TX, USA \\ Email: robert@shulerresearch.org
}

How to cite this paper: Shuler, R.L. (2021) Emergence of Objective Reality in an Irreversible Friend Thought Experiment. Journal of Applied Mathematics and Physics, 9 , 1964-1977.

https://doi.org/10.4236/jamp.2021.98128

Received: January 6, 2021

Accepted: August 22, 2021

Published: August 25, 2021

Copyright (อ 2021 by author(s) and Scientific Research Publishing Inc. This work is licensed under the Creative Commons Attribution International License (CC BY 4.0).

http://creativecommons.org/licenses/by/4.0/ (c) (i) Open Access

\begin{abstract}
Recently some photon models of a Wigner's friend experiment have led investigators to suggest that objective reality does not exist, and to publish articles with such claims. The public is not equipped to evaluate the severe limitations of these experiments. The separation of Wigner from the experiment and use of only reversible coherent processes for the friend allow operations that are not possible in ordinary reality according to the latest quantum research. We suggest directly testing the implied claim that objective reality, including incoherent objects with irreversible non-destructive memory, can be held in superposition. We suspect that it will fail, but provide for a graduated approach that may discover something about the conditions for superposition collapse. To this end, we design a thought experiment to model the objective world, investigating under what conditions experimenters in the same world (ensemble member) will be able to record a result and find it does not appear to change. This is not an investigation of hidden variables. To model the objective world, incoherent and irreversible processes must be included. To test for superposition, coherence has to be established. These seem to present a contradiction. The thought experiment suggests new places to look other than size for the origin of objective reality, casts doubt on the Many-Worlds interpretation, and provides a method of testing it.
\end{abstract}

\section{Keywords}

Quantum Measurement, Objective Reality, Wigner's Friend, Irreversibility, Waveform Collapse, Many-Worlds

\section{Introduction}

The early development of quantum theory was marked by debates over its completeness with respect to being able to fully describe the physical world. The Co- 
penhagen interpretation was thought to call into question scientific realism. Bohm's deterministic version of quantum mechanics, which violates locality, may have been partly disregarded because his leftist views got him an appearance before the House Committee on Un-American Activities after which he was ostracized. It was assumed by many that some threshold of complexity or size would trigger so-called waveform collapse, but by 1957 Everett proposed his Many-Worlds theory in which the probability wave never collapses [1], later given the catchy name by Bryce DeWitt [2]. Numerous summaries of this colorful history exist, such as Becker's What is Rea? [3]

Wigner, influenced by von Neumann's argument that the consciousness of an observer precipitates collapse [4], included observers in the measurement problem, reformulating Schrödinger's cat [5] to incorporate an observer known as Wigner's friend [6], later formalized by Deutsch [7].

By 1999 wave-particle duality was demonstrated for carbon 60 molecules [8]. These types of experiments continue, and if there is a "size" at which superposition disappears, it is not yet in evidence. In 2019 the superposition of a 2000 atom molecule was demonstrated, although the transverse displacement demonstrated was of the order of 1000 nanometers, a few times the size of the molecule [9].

In 2017, Brukner clarified some points in Deutsch's version of the Wigner's Friend thought experiment, and his is the starting point taken by more recent works. Two laboratories each measure one of a pair of entangled particles. They announce to a superobserver outside the lab whether they have a definite result, without revealing it. Brukner showed that this scenario has the problem of a hidden variable. One cannot assume fixed absolute facts between the superobserver (Wigner) and the observer in the lab (the friend). We take this as undisputed. Brukner's Extended Wigner Friend Scenario (EWFS) assumes that the superobservers can manipulate the quantum state of the laboratories, and derives certain inequalities they can measure to test observer independent facts (later described by other authors as absoluteness) [10].

We are not sure that the laboratories can be manipulated in that way, but if they can be, we take no issue with the result. The simpler proxies for "labs" in recent experiments can be manipulated in that way. Whether they model friends and labs is the question. Friends and labs include incoherent elements, and irreversible processes such as non-destructive readout memory (a memory which gives the same result on every readout or scan and is thus irreversible unlike a quantum observable).

Frauchiger and Renner argued in 2018 that quantum theory cannot consistently describe the use of itself, constructing a Gödel-like falsification [11]. This did not appear to deter experimenters, two groups of which have placed a pseudo-observer into an experiment in the manner of Wigner's friend and verified that certain correlation conditions are met. One uses six photons [12]. The observers in the experiments are not conscious of course, consisting of subatomic entities which may not have the freedom to activate the Frauchiger-Renner ar- 
gument. The next experiment, also using photons, is a proof of concept for a no-go theorem [13] which holds that one of the following propositions must be false:

1) There is no superdeterminism (preordained outcomes, deities selecting outcomes, etc.).

2) Physics is local (except for the most benign entanglement interactions).

3) Absoluteness of observed events (which if restricted to a world line we term "objectivity").

4) Quantum mechanics is as valid for "observers" as for anything else.

The first three are taken from Brukner's work, and the fourth specifically allows quantum mechanics to describe observers, placing the negative of Frauchiger and Renner's conclusion among the statements one of which must be false. Our investigation accepts as true propositions \#1 and \#2 above with respect to our proposed thought experiment, and will consider further Frauchiger-Renner in the discussion section. We primarily investigate the scope of objectivity, as to whether it requires collapse or exists meaningfully in superposition. This roughly replaces consideration \#3 in the above list. "Absoluteness" in the sense of [13] is too strong a term for us, since we consider superobservers unable to discuss specific results as not in the same world line as the observers in their experiments. If superpositions exist in the experiment, a world line is an objectively consistent state which is an element of the superposition.

All this is entwined with what an observer is or consists of. Clearly the experiments mentioned have taken a very limited view of the capabilities of an observer, only a photon or two, while von Neumann takes perhaps the most extreme position. No precise definition exists in quantum mechanics (though a definition of "observable" exists it is no help in this discussion).

The claim we wish to address, with a new thought experiment, is that "objective reality does not exist" [14]. This claim was made by some of the authors of [12]. What is objective reality? Do any of the EWFS experiments actually model it? "Extraordinary claims require extraordinary evidence," Carl Sagan said in his 1980 television show Cosmos.

While these experiment reports and theorems discuss "absoluteness of observed events," none presents two observers arguing over an event or whether it changed since being observed and recorded. In the EWFS the superobservers are only allowed to know that the lab observers have a definite result, not what it is. One of the operations permitted the superobservers in [13] is to erase the memory of the lab observers. That would only violate our definition of objectivity if it failed to also erase all records associated with those memories. Reversing a measurement if a non-destructive record is kept is thought not to be possible [15].

The purpose of this paper is to propose experiments that proactively address what we would ordinarily call objective reality. For this there must be at least two observer-viewers inside the lab who are allowed to discuss and disagree or agree about something objective. We have long accepted that what we think of as 
objective reality does not exist in the quantum world, and the question is under what conditions the objectivity property of the macroscopic world appears. We seek to learn from the thought experiment whether those inside a particular lab would ever confront non-objective conflicts of fact, and separately whether such labs can be proven to exist in superposition, or the minimum conditions at which they enter or exit superposition. A limitation of our approach is that we are not certain an ensemble of coherent quantum states can be obtained in a corresponding real world experiment. But if it cannot, then the point of the thought experiment is in any case established.

\section{Approach}

In a conventional experiment based on Wigner's Friend there is not one friend but an ensemble of them. As the external Wigner is required to perform some of the experimental duties but is not part of the ensembles, the experiment is an incomplete model of objective reality in which various investigators within the reality (i.e. within the experiment, if we are modeling reality) check for consistency.

We remedy this situation in two steps. First a measurement apparatus is defined as able to interact with a selected quantum object. The result of this interaction is that some information about the quantum state of the object (spin, polarity, position, momentum, etc.) is stored in the apparatus. Because of the no-cloning rule, the state of the quantum object is necessarily changed, but this does not concern us here.

The measurement apparatus stores the information obtained in a manner that can be interrogated more than once, for example as an exposed pixel on a photographic plate, some bits in the memory of a computer, a mark on a printout, etc. This is called the apparatus memory.

Second the stored information is "read" by another interaction which can be of any type, e.g. exposure to light, an electronic signal, etc. While this reading process is certainly composed of quantum transactions, they have energy transition thresholds that enable non-destructive reading, or at least copying from which the memory can be restored as well as passing on the information.

The information is read by a "viewer" which may be a human investigator, or some other apparatus, not necessarily capable of quantum measurement, but capable of performing the viewing interaction (or scan) and storing the result (in the viewer memory) and comparing the information stored to the information stored in another viewer or in the apparatus (in case of a second scanning).

At least one viewer is included in the experimental setup. Many viewers can be included, but at least one must be present. The viewer is tasked with scanning the apparatus repeatedly and verifying that the results stored in the measurement apparatus are consistent with its own memory of the previous scan. The viewer reports the results as "objectivity maintained" meaning the record of memory is consistent from scan to scan, or "objectivity violated" meaning a scan 
of the memory record conflicts with the viewer's independent record of prior scans.

Why introduce a quantum measurement at all if we are going to immediately try to destroy its superposition with an incoherent interaction? First of all the key word is "try." Second, we can vary the memory and scan interactions. We can use a coherent scan, or a partly coherent scan, and see if the superposition is truly destroyed. We can vary the threshold of readability of the memory.

Notice that we do not require any external observer to certify the results are consistent. The experiment, that is a single ensemble within it, must model the entirety of what the experiment defines as the objective reality it wishes to model. An external observer might see different stored information each time a scan is performed. But if the memory of the apparatus and the viewer change in sync, then the viewer might still report objectivity to be upheld. We do not think this likely because it violates locality. The two memories might be entangled, but they are not pure quantum states. They can be read and copied. A schematic is shown in Figure 1.

The experiment is to be performed in the usual way. An ensemble of these self-contained models of reality is produced, allowed to go through their internal measurement, recording, viewing, comparison and verdict issuing process, and correlations or interference are attempted to see if the results remain in superposition. Many variations are possible. Only the verdict might be tested for superposition. This might produce different results as the memory read-without-destruction threshold is varied and the coherence of the viewing scan is varied.

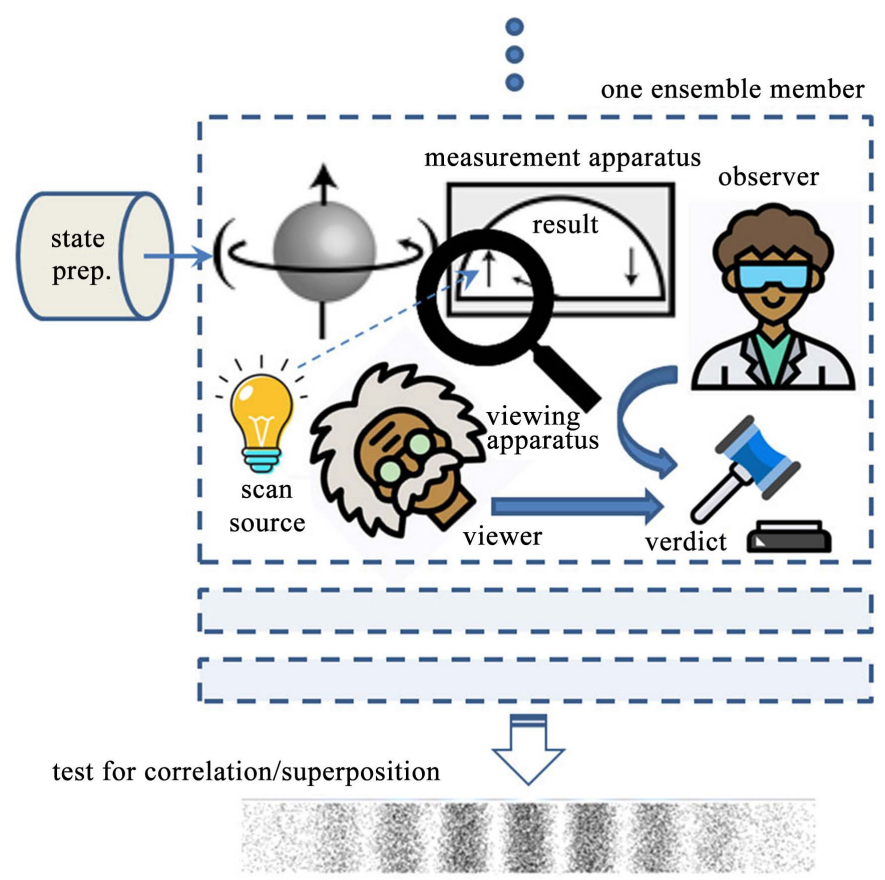

Figure 1. Thought experiment regarding the conditions for emergence of objective reality (Some icons from https://www.flaticon.com/). 
The purpose of the initial quantum measurement is to attempt to force the lab into superposition. The purpose of the memory scanning and viewing is twofold. First it allows the occupants of the lab to test for objectivity. Second it allows us, by varying the nature of the scan and memory, to examine conditions which force the lab out of superposition. The purpose of the correlation or interference test at the end is to see if the lab remains in superposition.

Consider the nature of the recording, scanning and comparison processes. At least one of the processes deliberately uses an interaction which is not correlated with the measurement interaction or the quantum state of the measured object. This might be a thermal photoflash, sunlight, or in the most universal case cosmic microwave background radiation. We make the specific hypothesis that once an incoherent or uncorrelated interaction is used on the recorded measurement information, in a non-destructive readout apparatus, then further scans will agree with the original scan. These scans are not quantum measurements, and so we avoid calling them "observations," but in the ordinary world we would say we observe the record of the measurement.

The nature of the measurement recording device, with non-destructive readout memory, is consistent with a suggestion by Sokolovski that instead of sentient observers, "participation in a measurement is limited to the changes produced in material objects, thus leaving her/her consciousness outside the picture" [16], a view widely supported [17].

\section{Results}

Demonstration of superposition for 2000 atom molecules appears to remove size alone as an obstacle to actually carrying out the proposed thought experiment. It seems likely that is enough to construct two memory devices, a means of measurement and a means of reading from one memory device to the other and comparing the result, with somewhat higher fidelity than using a few photons to model reality. In news releases the investigators involved have suggested a tenfold increase in size is imminent [18]. A few thousand nanometers of displacement might not support the Many-Worlds theory, but it certainly "might" test objective reality. The issue is no longer size but other factors such as decoherence and non-destructive memory, the two of which probably amount to irreversibility.

The use of an uncorrelated signal to transfer information upon which the objectivity verdict depends at first seems to imply that no correlations or interference patterns would be found past that point (unless they are newly introduced past that point), and so we as the meta-experimenters cannot find our ensembles correlated and cannot infer that any one of them remains in a superposition of multiple states. If the observer in the experiment was a Schrödinger cat, it would not be in superposition, even though the agent of collapse was entirely within "the box." Waveform collapse has occurred, by the terms of our experiment. That does not mean they could not possibly be in superposition, but that we cannot detect it, because as far as we know, in our accessible universe, we have 
used an uncorrelated signaling process.

If the "verdict" rendered by ALL members of the ensemble is that objectivity is upheld then we know that 1) our experiment models objective reality as far as we can tell, and 2) that these conditions are sufficient to support objectivity. We do not know if they are necessary, i.e. that there are no simpler conditions. We do not actually know whether it was the memory requirement or the incoherent signaling that established objective reality, though experimental variations might reveal this. To some degree decoherence is linked to irreversibility [19]. Irreversibility linkage to quantum thermodynamics has been experimentally confirmed [20].

If the verdict rendered by any member of any ensemble, beyond the expected experimental error rate, is that objectivity is not upheld, then we know that we have NOT successfully modeled objective reality and need to design a new experiment with new conditions.

However, coherence is relative to some sources. While there are certainly intrinsic tests for coherence, there is no guarantee coherence is not so fine-grained our experimental methods would not presently find it. Suppose there are two sets of oscillators, $i-j$ and $m-n$. Both are finely and erratically spaced so as to simulate an incoherent signal to our present methods of detection and analysis. Yet someone in possession of the original oscillators could likely prove the coherence of the signal. Suppose we construct the viewing signal using a set of such oscillators for the supposedly incoherent and uncorrelated readout scan. Then we use the original oscillators to test for superposition in the result. The author does not know what result we will get, but any result is new information and interesting.

If a real version of this experiment is constructed, we suppose it could be approached from two directions.

1) A weak version with a coherent scanning signal and some compromise in the memory apparatus (which we do not define) establishes that the framework of the experiment is correct and superposition can be achieved and detected.

2) A strong version with incoherent scan and non-destructive readout memory establishes a result that we can reasonably extrapolate to objective reality. If that result differs from the weak case, then we have the cause of the difference trapped between two experimental conditions and can converge on it.

To further investigate the Many-Worlds hypothesis, assuming a basically working experiment of at least the weak version, we propose an intermediate version using two ensembles, one called A using oscillators $i-j$ for supposedly incoherent signaling, and one called B using $m-n$. Now we call in two assistants, Wigner and Zeilinger, and give the $\mathbf{A}$ ensembles to Wigner and the $\mathbf{B}$ ensembles to Zeilinger and ask them to test for objective reality. Both might report a positive result, that objective reality is maintained. Then we could provide the matching oscillators to each assistant and they might be forced to reverse themselves. Thus the Many-Worlds interpretation might be valid but we might at 
present be unable to find confirmation. We might imagine mixed sets of oscillators and all sorts of variations.

\section{Discussion}

\subsection{What Would Schrödinger's Cat Say?}

Let us return for a moment to the old problem of Schrödinger's cat. First note that we have not alleviated the troublesome aspects of this problem. While the cat has a memory, Schrödinger carefully contained it in its own universe. The cat and any other occupants of its box may know whether it is alive or dead, or at least the observers in the box may find consistency even if they are in superposition, but outside the box we do not know, we are superobservers, not allowed to know. But is the result definite or a superposition of states? We claim that our proposed experiment tests this proposition. Our experimental apparatus with two recording non-destructive memories and readout signaling approximates a very simple cat. If there is a mouse also in the box, or even a flea, it certainly serves as the viewer. If we find no correlations either by interferometry or other methods among ensemble members, then there is only a single macro-state in the box.

The experiment also tests objectivity. Will the mouse and flea agree on whether the cat is alive or dead? We superobservers are irrelevant to this question. By analogy in the universe at large, when considering whether the entire observable universe is objective, there are no superobservers with which to consult (that we know of). Furthermore, once we have opened the box and disturbed the superposition, as far as objective reality is concerned, it only matters that the mouse, the flea and the cat all tell the same story. If there are multiple states superposed, objective reality only depends on not getting them mixed up.

\subsection{What Does Frauchiger-Renner Imply?}

Neither Schrödinger's cat nor our experiment with two memories, viewer and comparator, rise to the level of a quantum variable that has a conjugate whose measurement can be used to reinstate superposition. Frauchiger-Renner calls this evasively a "complex" measurement without describing how to do it. In fact it is key to "universal validity" of quantum mechanics.

The problem is illustrated by attempting to make a conjugate measurement on the cat. If it was in superposition and found upon measurement of its live-dead property to be dead, then it could, if it was a quantum variable, be restored to live-dead superposition by a conjugate measurement. However, what is the conjugate of live-dead? It is not a quantum property and there is not a conjugate (perhaps "identity" after a tongue-in-cheek fashion, just find another cat). If we cannot put a cat inside a braket, assert any operation we wish and get a result that agrees with experiment, then universality is not obtained and Frauchiger and Renner are right. The question we are trying to address is exactly when and why such non-quantum properties emerge. 


\subsection{When Does Quantum Reality Emerge?}

It is also instructive to ask the opposite, that is, when and why quantum properties emerge. An ordinary beam of light does not show interference. But pass it through double slits, or even past the edge of a barrier, and ripples emerge. The original light beam has a great many quantum states, and the barrier removes some or most of them. Quantum states can become observable because of this state reduction. They are (apparently) not observable when too many are present to distinguish them.

Photons, used in the two most recent experiments, have a very low rate of interaction with other photons, too low to say they have statistical mechanics over the time frame of the experiments. Letting them interact with thermal objects already spoils the experiments. Photons do not spontaneously decay unless they have a very large amount of energy (enough to produce a particle pair). They are not suitable for forming a readable memory apparatus. Even in the classical large scale theory of relativity, photons do not experience time.

If measurement truly collapses a wave function and results in a recorded fact, then objectivity is guaranteed by the collapse. If instead measurement passes the observer (and his lab) into a superposed state, then experiments such as [12] and [13] begin to seem to question objective reality, while our thought experiment recognizes the actual problem is how to maintain consistency in the complex superposition so that observers or viewers who may encounter each other are in possession of compatible facts. One could in principle hypothesize this directly which essentially was Everett's approach, but instead we have used a hypothesis very similar to some versions of the measurement hypothesis, deliberately so. In fact we have intimated it should be one of two things, relating to the non-destructive memory readout, or the use of a locally incoherent scanning interaction to interrogate memory or the measurement apparatus. Without the memory feature, objective reality would be pointless as there would be no means of detecting whether it was violated (locally, from within a worldline). With the incoherent scan, superposition cannot be detected because there is not coherence. If one suspects both these barriers are not necessary, our experiment is easily flexible on the matter of the scanning or memory mechanism. Scanning can be made partly or wholly coherent. Without the non-destructive memory, it amounts to something like the existing experiments. A quantum memory can be made reversible using work qubits to recover the prior state, but at increased risk of decoherence.

\subsection{Local Decoherence}

Other possibilities are considered in the literature on the measurement problem. For example there is the idea of continuous spontaneous localization (CSL) put forward by Ghirardi, Rimini and Pearle [21]. However, as Wechsler notes, "All these interpretations ignored the well known experimental fact that the reduction of the wave-function occurs in the presence of a macroscopic object and 
perturbs so many of its particles until its macroscopic state changes" [22].

How many irreversible state transitions does a C60 buckyball undergo during a quantum experiment? Is it a normal object? Is its small size what keeps its quantum properties, making it different from a cat which experiences time and entropy? Actually, no. It is the lockstep quantum bonds forming its structure. If it were simply a clump of 60 unbound atoms thermally evolving, no two clumps would evolve alike. Statistical mechanics produces entropy (and possibly therefore the arrow of time, and irreversibility). If an experiment attempts to store information permanently using a buckyball, what happens? Likely the information is stored in a quantum state which cannot be copied. If an irreversible change occurs, it isn't a buckyball anymore.

\subsection{The Possibility of Confirmation Bias}

It appears that due to the difficulty of preparing coherent states for quantum experiments, a kind of inadvertent confirmation bias has crept in. Only objects incapable of experiencing time and entropy are used in the experiments, and so results are devoid of irreversibility. This may exclude the most meaningful definitions of objectivity. Therefore our thought experiment posits that entropy experiencing objects (the readable memory, the incoherent scanning ray) must be included.

\subsection{Some Issues with Waves and Composite Objects}

Missing from quantum mechanics is an explanation of how a waveform selects a target for collapse, for example which atom should become excited, and how to conserve energy and momentum and meet all other relevant conditions. For the wave to purely represent a probability magnitude would be "nice" as an explanation, but superposition and entanglement foul that picture.

Waves are somewhat spurious to begin with, possibly another artifact of the development history. Schrödinger's wave mechanics was easier and more relatable to most people compared with Heisenberg's matrix mechanics. De Broglie's wave picture was less computationally capable than Schrödinger's, but retained knowledge that quantum objects are primarily oscillators, with no wave at all in their native reference frame, the wave being an artifact of relativistic time skew.

De Broglie's thesis was only translated into English in 2004 by Kracklauer [23]. There have been at least three English language papers rediscovering that de Broglie waves are derived from the Lorentz relation [24] [25] [26]. The author narrowly missed becoming a fourth when analyzing double slit experiments in moving reference frames [27]. In the rest frame of an electron, its de Broglie wave is completely synchronous in all space. It forms an interference pattern when a double slit and detector plate pass over it only because it is vertically deflected in interaction with the slits. Otherwise the wavelength would be infinite. If we assume a composite object "exists in reality" only when the de Broglie oscillations (not waves, it is not moving with respect to itself) of all its components in- 
terfere constructively, and neglect negative terms in the trig identity for multiplying sine functions, then we find the composite object de Broglie frequency is exactly the intermodulation product of the component frequencies [28].

This is an intriguing peek into massively composite quantum object behavior, far beyond what can be analyzed with current techniques. However, what works for mass may not work for spin or polarization. Spin direction does not add up, but rather averages out. If the component objects are not aligned in some regular way, spin is useless for measuring the superposition of a lab, or a cat. The only method the author knows of is to use the matter wave, that is, to throw an ensemble of labs or cats at a very small double slit. But the numbers that get through will be astronomically small. The failures in a quantum experiment are destroyed (see Wechsler [22]).

\subsection{Taking the Atom (Not the Cat) out of the Box}

If preparation of a quantum superposition by the method of a radioactive atom as suggested by Schrödinger has been demonstrated experimentally, the author cannot find it. It raises intriguing possibilities akin to entanglement. The atom could in principle be separated from the box without looking to see if it was decayed, or the box disturbed. Then if it has coupled to the contents of the box using superposition, not as a measurement event with waveform collapse, when does it uncouple?

This is similar to the coupling between entangled particles, and to the process of an electron or photon deciding which atom will be the target of absorption. We cannot at the present time envision productive ways of investigating this process, leading Everett to suggest that every possibility happens, similar to the rationale for the path integral. Methods of gradual spontaneous decoherence leave one wondering how the atom knows what is going on at the box and vice versa. A relativistic analysis finds that we cannot even set a logical order of which comes before the other.

\subsection{Disturbing Quantum Resonance, Non-Locally}

For discussion purposes, we introduce an idea similar to the Rutherford-Bohr atom, but non-local. The quantum object in the case of the radioactive atom is a bundle of energy to be emitted at some time. We propose it exists wherever it can find resonant support. Initially its wave function extends into the detector in the box, and not necessarily further as non-resonant blocking processes (incoherent or irreversible) exist beyond the front end of the detector. As long as it can be either there or in the atom, it is not compelled to decide, even if the atom is separated from the box. In the conventional view of measurement, when someone "looks" at the atom or in the box, it decides, but what is looking? That is something our thought experiment might discover.

Presumably something disturbs the resonant conditions enough that one or the other locations become non-viable, and the quantum object snaps to one 
place or the other. According to one analysis, even the loss of a single photon might prompt decoherence [29]. We suggest every collection of atoms and molecules that the waveform encompasses must be capable of accepting the superposition. If at any point it cannot, because it is not coherent or would enter irreversible quantum states, then the energy packet decides whether to remain with the atom or the detector in the box. The resonance which allowed the energy to be both here and there requires coherence, and a back and forth. Incoherence would disturb it, and irreversibility would trap it. And thus those are the two conditions chosen for investigation in our thought experiment.

\section{Conclusions}

We have shown that ensembles in a quantum experiment designed to model and test for objective reality as found in the large scale universe should contain at least two non-destructive memories and reading and comparing interactions, and further that at some point in these processes, either the memories or the signaling, uncorrelated signals should be introduced. This prohibits the detection of superposition and essentially contains elements of objective reality (the repeatedly readable memory devices). The roles of both Wigner and his friend are contained in the ensembles, i.e. in the experiment. In objective reality, we compare two readings of the same information source to determine consistency, not readings between our information sources and those in another world line. This definition of objective reality is consistent with philosophy of science deliberations such as Healey's [30].

The experiment should show that objective reality is maintained, since it contains elements of objective reality. In any case, the logical construction of this experiment shows that the prior experiments cited never should have confirmed objective reality in the sense we are implying (certainly they give valid results regarding hidden variables), as they didn't contain sufficient elements of objective reality.

Experiments were suggested using arrays of oscillators to simulate the incoherent parts of the experiment, which would provide tests in principle of the Many-Worlds interpretation, though we are not optimistic. Speculatively, it could be that measurement and viewing interaction entangle the state of interest with a target world. In the case of current-generation experiments, this is a very small coherent world, and an ensemble of them is demonstrated by the experiments. However, if irreversible processes alone spoil the superposition, we could reject Many-Worlds, along with the superposed version of Schrödinger's cat (which was what Schrödinger intended). For either to survive, conditions for objectivity must be less restrictive than for collapse of superposition. While it is well known that it is difficult to falsify Many-Worlds, claiming support for it from experiment must also open it to detraction from experiment.

In the discussion we present an alternative view of collapse as due to the limitations of non-local quantum resonance. Either decoherence or irreversibility 
would likely break up such resonance and relegate the energy quanta to a resonant subdivision of its former wave extent.

In summary, we have proposed a new thought experiment, with the goal of modeling the objective world, thus avoiding inadvertent confirmation bias. If the experiment cannot be implemented, trying to do so may uncover theoretical reasons. If the experiment can be implemented, it contains options for studying which kinds of phenomena (e.g. coherence or statistical mechanics or irreversibility, etc.) give rise to objective reality.

\section{Conflicts of Interest}

The author declares no conflicts of interest regarding the publication of this paper.

\section{References}

[1] Everett, H. (1957) Relative State Formulation of Quantum Mechanics. Reviews of Modern Physics, 29, 454-462. https://doi.org/10.1103/RevModPhys.29.454

[2] DeWitt, B.S. (1970) Quantum Mechanics and Reality. Physics Today, 23, 30-35. https://doi.org/10.1063/1.3022331

[3] Becker, A. (2018) What Is Real? Hachette Book Group, New York.

[4] Von Neumann, J. (1932) The Mathematical Foundations of Quantum Mechanics. English Translation by Beyer, R.T. Princeton University Press, Princeton.

[5] Schrödinger, E. (1935) The Present Situation in Quantum Mechanics. Naturwissenschaften, 23, 807-812. https://doi.org/10.1007/BF01491891

[6] Wigner, E.P. (1961) Remarks on the Mind-Body Question. In: Good, I.J., Ed., The Scientist Speculates, Heinemann, London.

[7] Deutsch, D. (1985) Quantum Theory as a Universal Physical Theory. International Journal of Theoretical Physics, 24, 1-41. https://doi.org/10.1007/BF00670071

[8] Arndt, M., Nairz, O., Vos-Andreae, J., Keller, C., vand der Zouw, G. and Zeilinger, A. (1999) Wave-Particle Duality of C60 Molecules. Nature, 401, 680-682. https://doi.org/10.1038/44348

[9] Fein, Y.Y., Geyer, P., Zwick, P., Kialka, F., Pedalino, S., Mayor, M., Gerlich, S. and Arndt, M. (2019) Quantum Supperposition of Molecules beyond $25 \mathrm{kDa}$. Nature Physics, 15, 1242-1245. https://doi.org/10.1038/s41567-019-0663-9

[10] Brukner, C. (2017) On the Quantum Measurement Problem. Quantum Physics, arXiv:1507.05255.

[11] Frauchiger, D. and Renner, R. (2018) Quantum Theory Cannot Consistently Describe the Use of Itself. Nature Communications, 9, Article No. 3711. https://doi.org/10.1038/s41467-018-05739-8

[12] Proietti, M., Pickston, A., Graffitti, F., Barrow, P., Kundys, D., Branciard, C., et al. (2019) Experimental Test of Local Observer Independence. Science Advances, 5, Article ID: eaaw9832. https://doi.org/10.1126/sciadv.aaw9832

[13] Bong, K., Utreras-Alarcon, A., Ghafari, F., Liang, Y., Tischler, N., Cavalcanti, E.G., Pryde, G. and Wiseman, H.M. (2020) A Strong No-Go Theorem on the Wigner's Friend Paradox. Nature Physics, 16, 1199-1205. https://doi.org/10.1038/s41567-020-0990-x

[14] Fedrizzi, A. and Proietti, M. (2019) Objective Reality Doesn’t Exist, Quantum Expe- 
riment Shows. LiveScience.

https://www.livescience.com/objective-reality-not-exist-quantum-physicists.html

[15] Zurek, W.H. (2018) Quantum Reversibility Is Relative, or Does a Quantum Measurement Reset Initial Conditions? Philosophical Transactions of the Royal Society $A$, 376. https://doi.org/10.1098/rsta.2017.0315

[16] Sokolovski, D. (2020) Quantum Measurements with, and Yet without an Observer. Entropy, 22, 1185. https://doi.org/10.3390/e22101185

[17] Snyder, D.M. (2000) Irreversibility and Measurement in Quantum Mechanics.

[18] Letzter, R. (2019) 2000 Atoms Exist in Two Places at Once in Unprecedented Quantum Experiment. LiveScience. https://www.livescience.com/2000-atoms-in-two-places-at-once.html

[19] Mohammady, M.H., Auffeves, A. and Anders, J. (2020) Energetic Footprints of Irreversibility in the Quantum Regime. Communications Physics, 3, Article No. 89. https://doi.org/10.1038/s42005-020-0356-9

[20] Peterson, J.P.S., Batalhão, T.B., Herrera, M., Souza, A.M., Sarthour, R.S., Oliveira, I.S. and Serra, R.M. (2019) Experimental Characterization of a Spin Quantum Heat Engine. Physical Review Letters, 123, Article ID: 240601. https://doi.org/10.1103/PhysRevLett.123.240601

[21] Ghirardi, G., Pearle, P. and Rimini, A. (1990) Markov Processes in Hilbert Space and Continuous Spontaneous Localization of Systems of Identical Particles. Physical Review A, 42, 78-89. https://doi.org/10.1103/PhysRevA.42.78

[22] Wechsler, S.D. (2020) In Praise and in Criticism of the Model of the Continuous Spontaneous Localization of the Wave-Function. Journal of Quantum Information Science, 10, 73-103. https://doi.org/10.4236/jqis.2020.104006

[23] De Broglie, L. (1925) On the Theory of Quanta. Annales de Physique, 10. https://aflb.minesparis.psl.eu/LDB-oeuvres/De Broglie Kracklauer.pdf https://doi.org/10.1051/anphys/192510030022

[24] Baylls, W.E. (2007) De Broglie waves as an effect of clock desynchronization. Canadian Journal of Physics, 85, 1317-1323. https://doi.org/10.1139/p07-121

[25] Dogra, R. (2002) A New Proposal Combining Quantum Mechanics and the Special Theory of Relativity. Aperion, 9, 20-27.

[26] Ylmaz, H. (2010) Lorentz Transformations and Wave-Particle Unity. Physics Essays, 23, 334-336. https://doi.org/10.4006/1.3409115

[27] Shuler, R.L. (2015) Common Pedagogical Issues with De Broglie Waves: Moving Double Slits, Composite Mass, and Clock Synchronization. Physical Sciences Research International, 2015, Article ID: 895134. https://doi.org/10.1155/2015/895134

[28] Wignall, J.W.G. (1985) De Broglie Waves and the Nature of Mass. Foundations of Physics, 15, 207-227. https://doi.org/10.1007/BF00735293

[29] Serafini, A., De Siena, S., Illuminati, F. and Paris, M.G.A. (2003) Minimum Decoherence Cat-Like States in Gaussian Noisy Channels. Journal of Optics B, 6, S591. https://doi.org/10.1088/1464-4266/6/6/019

[30] Healey, R. (2018) Quantum Theory and the Limits of Objectivity. Foundations of Physics, 48, 1568-1569. https://doi.org/10.1007/s10701-018-0216-6 